Алгебра и анализ

Том 20 (2008), № 5
St. Petersburg Math. J.

Vol. 20 (2009), No. 5, Pages 837-849

S 1061-0022(09)01075-9

Article electronically published on July 21, 2009

\title{
ON GENERALIZED WINDING NUMBERS
}

\author{
V. V. CHERNOV (TCHERNOV) AND Y. B. RUDYAK
}

\begin{abstract}
Let $M^{m}$ be an oriented manifold, let $N^{m-1}$ be an oriented closed manifold, and let $p$ be a point in $M^{m}$. For a smooth map $f: N^{m-1} \rightarrow M^{m}, p \notin \operatorname{Im} f$, an invariant $\operatorname{awin}_{p}(f)$ is introduced, which can be regarded as a generalization of the classical winding number of a planar curve around a point. It is shown that $\operatorname{awin}_{p}$ estimates from below the number of passages of a wave front on $M$ through a given point $p \in M$ between two moments of time. The invariant awin $\operatorname{makes~it~}$ possible to formulate an analog of the complex analysis Cauchy integral formula for meromorphic functions on complex surfaces of genus exceeding one.
\end{abstract}

\section{INTRODUCTION}

The Gauss linking number is a link homotopy invariant of a pair $\left(\phi_{1}\left(N_{1}^{n_{1}}\right), \phi_{2}\left(N_{2}^{n_{2}}\right)\right)$ of disjoint linked closed oriented submanifolds in an oriented manifold $M^{m}$ of dimension $m=n_{1}+n_{2}+1$. The linking number $\mathrm{lk}$ is defined via basic homology theory as the intersection number of a singular chain whose boundary is $\phi_{2}\left(N_{2}\right)$ with $\phi_{1}\left(N_{1}\right)$.

If $\phi_{2 *}\left(\left[N_{2}\right]\right) \neq 0 \in H_{*}(M)$, then $\phi_{2}\left(N_{2}\right)$ is not a boundary of any singular chain. If $\phi_{2 *}\left(\left[N_{2}\right]\right)=0$ but $\phi_{1 *}\left(\left[N_{1}\right]\right) \neq 0 \in H_{*}(M)$, then the intersection number in the definition above does depend on the relative homology class in $H_{*}\left(M, \phi_{2}\left(N_{2}\right)\right)$ realized by the singular chain. Thus $\mathrm{lk}$ is not well defined unless the link components are homologous to zero. (Similar homology theory methods allow one to define the linking number in the case where $\phi_{i *}\left(\left[N_{i}\right]\right) \in H_{*}(M)$ are of finite order or $\phi_{i *}\left(\left[N_{i}\right]\right)=0 \in H_{*}(M, \partial M), i=1,2$; see Kaiser [11.)

The winding number $\operatorname{win}_{p} f$ of a loop $f: S^{1} \rightarrow \mathbb{R}^{2}$ around a point $p \notin \operatorname{Im} f$ measures how many times $f$ turns around $p$. It is defined as the intersection number of a path $P$ connecting $p$ to infinity with a loop $f$. Clearly the winding number is simply the linking number between $f: S^{1} \rightarrow S^{2}=\mathbb{R}^{2} \cup\{\infty\}$ and the map of $S^{0}$ sending one point of $S^{0}$ to $p$ and the other to $\infty$.

The winding number is a classical invariant. It is a part of the complex analysis Cauchy integral formula and it has many applications in topology. The result of Whitney [21] expresses the rotation number of a planar curve through the winding numbers of the curve around points in the regions of the curve complement. Many formulas involving the winding number for invariants of planar curves, fronts, and knot diagrams were obtained in the work of Polyak [13, Shumakovich [16, 17, Turaev [18, Viro [20], and the first author 4]. Generalizations of winding numbers to hypersurfaces in $\mathbb{R}^{m}$ are essential in the work of Goryunov [10, Mikhalkin and Polyak [12.

Since the winding number is a particular case of the linking number, standard homology theory methods give its generalization to the case of a (possibly singular) smooth hypersurface $f: N^{m-1} \rightarrow M^{m}, p \notin \operatorname{Im} f$, provided that $f_{*}([N])=0 \in H_{m-1}(M)$ and

2000 Mathematics Subject Classification. Primary 55M25; Secondary 53Z05, 57R35.

Key words and phrases. Affine winding number, linking number, invariant. 
that $\partial M \neq \varnothing$ or that $M$ is the interior of a manifold with boundary. (A boundary component plays the role of infinity where one places the second point of $S^{0}$. The condition $f_{*}([N])=0$ is needed, so that the intersection number of the path $P$ and the hypersurface $f(N)$ be independent of the choice of the path $P$.) We denote the winding number defined in this way by $\operatorname{win}_{p}(f)$.

In our paper [6], we constructed the "affine linking invariant" alk of a pair of linked singular oriented closed submanifolds $\left(\phi_{1}\left(N_{1}^{n_{1}}\right), \phi_{2}\left(N_{2}^{n_{2}}\right)\right)$ of $M^{n_{1}+n_{2}+1}$. Our alk is a link homotopy invariant. It is a generalization of the linking number and it is well defined for all $\phi_{1 *}\left(\left[N_{1}\right]\right), \phi_{2 *}\left(\left[N_{2}\right]\right) \in H_{*}(M)$. The group where the alk invariant takes values depends on the homotopy classes of the maps $\phi_{1}, \phi_{2}$ and sometimes is hard to compute.

In this paper we use ideas similar to those used in [6] to define alk, to construct the affine winding number $\operatorname{awin}_{p}(f)$ that is a generalization of the winding number to a vast collection of oriented $M$ and closed oriented $N$. We do not require that $f_{*}([N])=0 \in$ $H_{*}(M)$ and that $\partial M \neq \varnothing$ (or that $M$ be the interior of a manifold with boundary). Thus, in these cases the invariant $\operatorname{win}_{p}(f)$, which is a particular case of the linking number, is not defined.

Since one of the linked manifolds is a one-point space, many of the technical difficulties we dealt with in [6] do not arise. In particular, the group where the affine winding number takes values is either $\mathbb{Z}$ or a quotient group of $\mathbb{Z}$. Also, the operation $\mu$ on the bordism groups, which we introduced in 6 to define alk, reduces to the standard intersection pairing. (The operation $\mu$ is quite interesting on its own. It gives rise to a Poisson bracket on the bordism group of garlands, see [7, 5, which is related to Goldman-Turaev [9, 19] and Andersen-Mattes-Reshetikhin 1, 2 algebras.)

The generalized affine winding numbers we construct in this paper have an affine nature; i.e., only the difference of affine winding numbers of two homotopic maps is well defined. Equivalently, for a fixed distinguished $\varepsilon: N \rightarrow M$, we can define the affine winding number $\operatorname{awin}_{p}(f)$ of $f: N \rightarrow M$ around $p$ provided that $f$ and $\varepsilon$ are homotopic. In the classical case of $M=\mathbb{R}^{2}$ and $N=S^{1}$, such a distinguished map is, in a sense, fixed implicitly, and it is a map into a point in $\mathbb{R}^{2} \backslash p$. The precise definitions are given in $\$ 1$.

In $₫ 2$ we consider two applications of our theory. First, we formulate a generalization of the complex analysis Cauchy integral formula to the case of meromorphic functions on complex surfaces of genus exceeding one. Second, we consider propagation of a wave front in $M$ and estimate the number of passages of the front through a given point between two time moments $t_{1}$ and $t_{2}$. In many cases we can estimate this number merely on the basis of the shapes of the front at time moments $t_{1}$ and $t_{2}$, without any knowledge of the propagation process, topology of $M$, etc.

In $\$ 3$ we generalize the invariant $\operatorname{awin}_{p}(f)$ to the case where the point $p \in M$ is not fixed and somehow moves in $M$.

\section{§1. Affine Winding NUmbers}

We work in the $C^{\infty}$-category, and the word smooth means $C^{\infty}$. In this paper $N^{m-1}$ and $M^{m}$ are oriented connected smooth manifolds of dimensions $m-1$ and $m$, respectively, and we assume that $m \geq 2$. The manifold $N$ is assumed to be closed. $I$ is the interval $[0,1]$.

In this section we fix a point $p \in M$ and a connected component $\mathcal{N}$ of the space $C^{\infty}(N, M)$ of smooth maps $N \rightarrow M$. Put $\Sigma \subset \mathcal{N}$ to be the discriminant that consists of all maps $f \in \mathcal{N}$ such that $p \in \operatorname{Im} f$. We do not include into $\Sigma$ the maps $f$ that are singular in the common sense but do not satisfy $p \in \operatorname{Im} f$. 
The affine winding number $\operatorname{awin}_{p}(f)$ that we define in this section is a locally constant function on $\mathcal{N} \backslash \Sigma$. Equivalently, it is a function on $\pi_{0}(\mathcal{N} \backslash \Sigma)$. If $f_{*}([N])=0 \in H_{*}(M)$ and $\partial M \neq \varnothing$, so that $\operatorname{win}_{p}(f)$ can be defined as the particular case of the linking number, then the functions $\operatorname{win}_{p}(f), \operatorname{awin}_{p}(f): \mathcal{N} \backslash \Sigma \rightarrow \mathbb{Z}$ are equal up to an additive constant.

1.1. Definition. Let $p$ be a point in $M$. We say that a smooth map $F: N \times I \rightarrow M$ is good if $p \notin F(N \times\{0,1\})$ and $p$ is a regular value of $F$. We also call such an $F$ a good homotopy between the maps $\left.F\right|_{N \times 0},\left.F\right|_{N \times 1} \in \mathcal{N} \backslash \Sigma$.

If two smooth maps $N \rightarrow M$ are homotopic and their images do not contain $p$, then there exists a good homotopy between these maps. Moreover, the set of good homotopies is $C^{0}$-dense in the set of all homotopies. This can be proved via standard general position arguments.

Note that $F^{-1}(p)$ is a finite set for every good $F$. The standard orientation of $I$ yields an orientation of $N \times I$. Every point in $F^{-1}(p)$ is equipped with a sign \pm 1 as follows. We put the sign of the point to be +1 if the restriction of $F$ to a small neighborhood of the point is orientation preserving, and we put the sign of the point to be -1 otherwise.

1.2. Definition. For a good map $F: N \times I \rightarrow M$, we define $\Delta_{\text {awin }_{p}}(F) \in \mathbb{Z}$ to be the sum of the signs of the inverse images of $p$ under $F$.

Remark (relationship between $\Delta_{\text {awin }_{p}}(F)$ and the intersection number). The number $\Delta_{\text {awin }_{p}}(F)$ can also be described as follows. Consider the maps

$$
\Phi: N \times I \rightarrow M \times I, \quad \Phi(x, t)=(F(x), t)
$$

and

$$
P: I \rightarrow M \times I, \quad P(t)=(p, t) .
$$

Then $\Delta_{\operatorname{awin}_{p}}(F)$ is equal to the intersection number of $\Phi$ and $P$. The proof is straightforward.

Similarly, $\Delta_{\text {awin }_{p}}(F)$ equals the intersection number of $F$ and of the positively oriented point $p$.

We regard the circle $S^{1}$ as the quotient space $I /\{0,1\}$ and denote by $\pi: I \rightarrow S^{1}$ the projection. Consider a good map $F: N \times I \rightarrow M$ and assume that $F(x, 0)=F(x, 1)$ for all $x \in N$. Then there exists a unique map $G: N \times S^{1} \rightarrow M$ with $F=G \circ\left(1_{N} \times \pi\right)$. The following lemma follows immediately from Definition 1.2 and the description of the degree as the sum of the signs of the inverse images. Note that if the manifold $M$ is not closed, then the degree of $G$ is zero by definition.

1.3. Lemma. $\Delta_{\text {awin }_{p}}(F)$ is equal to the degree of the map $G$.

1.4. Definition $(\mathcal{A}(M, \mathcal{N}))$. We say that a smooth map $\mu: N \times S^{1} \rightarrow M$ is special if $\left.\mu\right|_{N \times s} \in \mathcal{N}$ for some (and therefore for all) $s \in S^{1}$. We define the indeterminacy subgroup $\boldsymbol{A}=\boldsymbol{A}(M, \mathcal{N})$ of $\mathbb{Z}$ to be the subgroup of possible degrees of the special maps $\mu: N \times S^{1} \rightarrow M$. Put $\mathcal{A}(M, \mathcal{N})=\mathbb{Z} / \boldsymbol{A}$ and denote by $q: \mathbb{Z} \rightarrow \mathcal{A}(M, \mathcal{N})$ the quotient homomorphism.

1.5. Definition. Fix $\varepsilon=\varepsilon_{\mathcal{N}} \in \mathcal{N} \backslash \Sigma$, which should be thought of as a preferred map. Given a map $f \in \mathcal{N} \backslash \Sigma$, we define the affine winding number $\operatorname{awin}_{p}(f)=\operatorname{awin}_{p, \varepsilon}(f) \in$ $\mathcal{A}(M, \mathcal{N})$ by setting

$$
\operatorname{awin}_{p}(f)=q\left(\Delta_{\operatorname{awin}_{p}}(F)\right) \in \mathcal{A}(M, \mathcal{N}),
$$

where $F: N \times I \rightarrow M$ is a good homotopy between $\varepsilon=\left.F\right|_{N \times 0}$ and $f=\left.F\right|_{N \times 1}$.

Note that $\operatorname{awin}_{p, \varepsilon}(\varepsilon)=0$. 
1.6. Theorem. The invariant awin has the following properties:

1. The number $\operatorname{awin}_{p}(f)=\operatorname{awin}_{p, \varepsilon}(f) \in \mathcal{A}(M, \mathcal{N})$ does not depend on the choice of the good homotopy $F$ in its definition. Thus, $\operatorname{awin}_{p}: \mathcal{N} \backslash \Sigma \rightarrow \mathcal{A}(M, \mathcal{N})$ is well defined.

2. If $H$ is a good homotopy between $f_{0}=\left.H\right|_{N \times 0}$ and $f_{1}=\left.H\right|_{N \times 1}$, then $\operatorname{awin}_{p}\left(f_{1}\right)-$ $\operatorname{awin}_{p}\left(f_{0}\right)=q\left(\Delta_{\operatorname{awin}_{p}}(H)\right)$. In particular, $\operatorname{awin}_{p}: \mathcal{N} \backslash \Sigma \rightarrow \mathcal{A}(M, \mathcal{N})$ is a locally constant function.

3. If we replace $\varepsilon$ by some $\varepsilon^{\prime} \in \mathcal{N} \backslash \Sigma$, then $\operatorname{awin}_{p, \varepsilon^{\prime}}-\operatorname{awin}_{p, \varepsilon}: \mathcal{N} \backslash \mathbb{Z} \rightarrow \mathcal{A}(M, \mathcal{N})$ is the constant function $\operatorname{awin}_{p, \varepsilon^{\prime}}(\varepsilon)$. Hence the functions $\operatorname{awin}_{p, \varepsilon^{\prime}}, \operatorname{awin}_{p, \varepsilon}: \mathcal{N} \backslash \mathbb{Z} \rightarrow$ $\mathcal{A}(M, \mathcal{N})$ are equal up to an additive constant.

Proof. We prove statement 1. Let $F_{1}, F_{2}: N \times I \rightarrow M$ be two good homotopies between $\varepsilon$ and $f$. We must show that $q\left(\Delta_{\text {awin }_{p}}\left(F_{1}\right)\right)=q\left(\Delta_{\operatorname{awin}_{p}}\left(F_{2}\right)\right) \in \mathcal{A}(M, \mathcal{N})$. Define $\bar{F}_{2}$ : $N \times I \rightarrow M$ by $\bar{F}_{2}(n, t)=F_{2}(n, 1-t)$. Clearly, $\Delta_{\operatorname{awin}_{p}}\left(\bar{F}_{2}\right)=-\Delta_{\operatorname{awin}_{p}}\left(F_{2}\right)$. Thus, it suffices to show that $q\left(\Delta_{\operatorname{awin}_{p}}\left(F_{1}\right)+\Delta_{\operatorname{awin}_{p}}\left(\bar{F}_{2}\right)\right)=0 \in \mathcal{A}(M, \mathcal{N})$.

A homotopy $F_{1}$ followed by $\bar{F}_{2}$ gives a homotopy $F: N \times I \rightarrow M$ defined via $F(n, t)=F_{1}(n, 2 t)$ for $n \in N, t \in\left[0, \frac{1}{2}\right]$, and via $F(n, t)=\bar{F}_{2}(n, 2 t-1)$ for $n \in N$, $t \in\left[\frac{1}{2}, 1\right]$. Perturbing $F$ slightly in $N \times(0,1)$ if needed, we can assume that it is smooth and hence that $F$ is a good homotopy between $\left.F\right|_{N \times 0}=\varepsilon$ and $\left.F\right|_{N \times 1}=\varepsilon$. Clearly, $\Delta_{\text {awin }_{p}}(F)=\Delta_{\text {awin }_{p}}\left(F_{1}\right)+\Delta_{\text {awin }_{p}}\left(F_{2}\right)$. Lemma 1.3 implies that $q\left(\Delta_{\text {awin }_{p}}(F)\right)=$ $q\left(\Delta_{\operatorname{awin}_{p}}\left(F_{1}\right)+\Delta_{\operatorname{awin}_{p}}\left(\bar{F}_{2}\right)\right)=0 \in \mathcal{A}(M, \mathcal{N})$. Thus, the function $\operatorname{awin}_{p}: \mathcal{N} \backslash \Sigma \rightarrow$ $\mathcal{A}(M, \mathcal{N})$ is well defined.

To prove statement 2 , choose a good homotopy $F: N \times I \rightarrow M$ from $\left.F\right|_{N \times 0}=\varepsilon$ to $\left.F\right|_{N \times 1}=f_{0}$. Much as above, $F$ followed by $H$ gives a good homotopy $J$ from $\varepsilon$ to $f_{1}$. Clearly, $\Delta_{\operatorname{awin}_{p}}(J)=\Delta_{\operatorname{awin}_{p}}(F)+\Delta_{\operatorname{awin}_{p}}(H)$. By the definition of $\operatorname{awin}_{p}$, we have $\operatorname{awin}_{p}\left(f_{1}\right)=q\left(\Delta_{\operatorname{awin}_{p}}(J)\right)$ and $\operatorname{awin}_{p}\left(f_{0}\right)=q\left(\Delta_{\operatorname{awin}_{p}}(F)\right)$. Thus, $\operatorname{awin}_{p}\left(f_{1}\right)-\operatorname{awin}_{p}\left(f_{0}\right)=$ $q\left(\Delta_{\operatorname{awin}_{p}}(J)-\Delta_{\operatorname{awin}_{p}}(F)\right)=q\left(\Delta_{\operatorname{awin}_{p}}(H)\right)$.

If $f_{0}$ and $f_{1}$ belong to the same path connected component of $\mathcal{N} \backslash \Sigma$, then we can find a good homotopy $H$ between them such that $H^{-1}(p)=\varnothing$. Thus, $\operatorname{awin}_{p}\left(f_{1}\right)-\operatorname{awin}_{p}\left(f_{0}\right)=$ $q(0)=0$, and hence $\operatorname{awin}_{p}$ is a locally constant function.

We prove statement 3 . Clearly $\mathcal{A}(M, \mathcal{N})$ and $q: \mathbb{Z} \rightarrow \mathcal{A}(M, \mathcal{N})$ do not depend on the choice of $\varepsilon \in \mathcal{N} \backslash \Sigma$. Choose a good homotopy $F^{\prime}: N \times I \rightarrow M$ from $\varepsilon^{\prime}$ to $\varepsilon$. Choose $f \in \mathcal{N} \backslash \Sigma$ and a good homotopy $F$ from $\varepsilon$ to $f$. We have $\operatorname{awin}_{p, \varepsilon}(f)=q\left(\Delta_{\operatorname{awin}_{p}}(F)\right)$. As above, the homotopy $F^{\prime}$ followed by $F$ gives a good homotopy from $\varepsilon^{\prime}$ to $f$. Counting the preimages of $p$ under this homotopy, we see that $\operatorname{awin}_{p, \varepsilon^{\prime}}(f)=q\left(\Delta_{\text {awin }_{p}}(F)\right)+$ $q\left(\Delta_{\operatorname{awin}_{p}}\left(F^{\prime}\right)\right)$. Thus, $\operatorname{awin}_{p, \varepsilon^{\prime}}(f)-\operatorname{awin}_{p, \varepsilon}(f)=q\left(\Delta_{\operatorname{awin}_{p}}\left(F^{\prime}\right)\right)=\operatorname{awin}_{p, \varepsilon^{\prime}}(\varepsilon)$.

Remark (affine nature of $\operatorname{awin}_{p}$ ). By statement 3 of Theorem 1.6, if we change the distinguished map $\varepsilon \in \mathcal{N} \backslash \Sigma$, then the function $\operatorname{awin}_{p}=\operatorname{awin}_{p, \varepsilon}: \mathcal{N} \backslash \Sigma \rightarrow \mathcal{A}(M, \mathcal{N})$ changes by an additive constant. Thus, if we neglect the distinguished map $\varepsilon$, then the invariant $\operatorname{awin}_{p}$ is well defined up to an additive constant. This is similar to the ambiguity in the choice of the origin in an affine space, and this shows the affine nature of our invariant $\operatorname{awin}_{p}$.

Note also that statement 2 of Theorem 1.6 implies that for $f_{0}, f_{1} \in \mathcal{N} \backslash \Sigma$ the difference $\operatorname{awin}_{p, \varepsilon}\left(f_{1}\right)-\operatorname{awin}_{p, \varepsilon}\left(f_{0}\right)$ does not depend on the choice of the distinguished map $\varepsilon$.

It is useful to know when the indeterminacy subgroup $\boldsymbol{A}$ is trivial, i.e., $\mathcal{A}(M, \mathcal{N})=\mathbb{Z}$. For such spaces, as in the classical case of $M=\mathbb{R}^{2}$ and $N=S^{1}$, the affine winding numbers are indeed integers rather than elements of $\mathcal{A}(M, \mathcal{N})=\mathbb{Z} / \boldsymbol{A}$. 
1.7. Theorem. The identity $\mathcal{A}(M, \mathcal{N})=\mathbb{Z}$ occurs if and only if all the special mappings $N \times S^{1} \rightarrow M^{m}$ have zero degrees. In particular, $\mathcal{A}(M, \mathcal{N})=\mathbb{Z}$ provided that at least one of the following conditions is satisfied:

0. The manifold $M^{m}$ is not closed.

1. There exists $i \in\{1, \ldots, m\}$ such that $b_{i}(N)+b_{i-1}(N)<b_{i}(M)$, where $b_{i}$ is the ith Betti number.

2. The space $\mathcal{N}$ consists of null-homotopic maps and there exists $i \in\{1, \ldots, m\}$ such that $b_{i-1}(N)<b_{i}(M)$. (In particular, this condition is fulfilled if $b_{1}(M)>1$, because $N$ is connected.)

3. $N=S^{m-1}$, the space $\mathcal{N}$ consists of null homotopic maps, and $M$ is not a rational homology sphere.

4. A map from $\mathcal{N}$ induces the trivial homomorphism $\pi_{1}(N) \rightarrow \pi_{1}(M)$, the group $\pi_{1}(M)$ is infinite and it does not contain $\mathbb{Z}$ as a finite index subgroup.

5. $M$ is a closed manifold that admits a complete Riemannian metric of negative sectional curvature.

Proof. Clearly, $\mathcal{A}(M, \mathcal{N})=\mathbb{Z}$ if and only if all special maps $\mu: N \times S^{1} \rightarrow M$ have zero degrees. Thus, it suffices to show that if any one of Conditions $0-5$ is satisfied, then every special mapping $\mu: N \times S^{1} \rightarrow M$ has zero degree. We deal with each condition separately.

Condition 0. The degree of a map from a closed $m$-dimensional manifold to a nonclosed $m$-dimensional manifold is always zero. Thus, every special $\mu: N \times S^{1} \rightarrow M$ has zero degree.

For this reason, while considering the cases of Conditions 1-5, we can and shall assume that $M$ is closed.

Condition 1. Consider a special map $\mu: N \times S^{1} \rightarrow M$ of degree $l \neq 0$. We must prove that $b_{i}(N)+b_{i-1}(N) \geq b_{i}(M)$ for all $i$.

Let $\mu_{\text {! }}: H_{*}(M) \rightarrow H_{*}\left(N \times S^{1}\right)$ be the transfer map; see, e.g., [15, V.2.11]. As is well known, $\mu_{*} \circ \mu_{!}(x)=l x$ for all $x \in H_{*}(M)$. In particular, $\mu_{*}: H_{*}\left(N \times S^{1} ; \mathbb{Q}\right) \rightarrow H_{*}(M ; \mathbb{Q})$ is an epimorphism. Hence, $\operatorname{rank} H_{i}\left(N \times S^{1}\right)=b_{i}\left(N \times S^{1}\right) \geq \operatorname{rank} H_{i}(M)=b_{i}(M)$ for all $i=1, \ldots, m$. By the Künneth formula, we have $b_{i}\left(N \times S^{1}\right)=b_{i}(N)+b_{i-1}(N)$. Thus, $b_{i}(N)+b_{i-1}(N) \geq b_{i}(M)$ for all $i$.

Condition 2. Consider a special map $\mu: N \times S^{1} \rightarrow M$ of degree $l \neq 0$. We must prove that $b_{i-1}(N) \geq b_{i}(M)$ for all $i$.

Much as in the case of Condition 1 , we conclude that $\mu_{*}: H_{*}\left(N \times S^{1} ; \mathbb{Q}\right) \rightarrow H_{*}(M ; \mathbb{Q})$ is an epimorphism. Fix a point $* \in S^{1}$ and denote by $i: N \rightarrow N \times S^{1}, i(n)=(n, *)$ the inclusion. Consider a diagram of homology groups

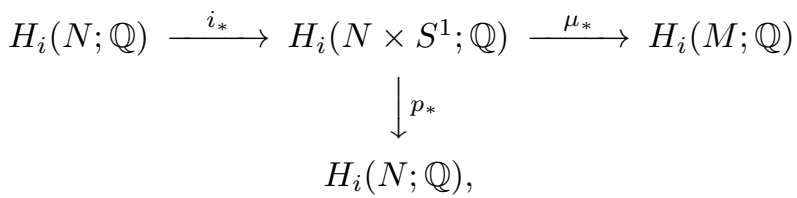

where $p: N \times S^{1} \rightarrow N$ is the projection.

Since $\mathcal{N}$ consists of null-homotopic maps, $\mu_{*} i_{*}$ is the zero homomorphism. Since $p_{*} i_{*}=\mathrm{id}$, we see that $i_{*}$ is injective, whence rank ker $\mu_{*} \geq b_{i}(N)$. Since $\mu_{*}$ is surjective, $\operatorname{rank} \operatorname{Im} \mu_{*}=b_{i}(M)$. Using the Künneth formula and elementary linear algebra, we obtain $b_{i}(N)+b_{i-1}(N)=b_{i}\left(N \times S^{1}\right)=\operatorname{rank} \operatorname{ker} \mu_{*}+\operatorname{rank} \operatorname{Im} \mu_{*} \geq b_{i}(N)+b_{i}(M)$. Thus, we have $b_{i-1}(N) \geq b_{i}(M)$. 
Condition 3. Assume that $\operatorname{dim} M=m>2$. If $M$ is not a rational homology sphere, then there exists $i$ with $1 \leq i \leq m-1$ and such that $b_{i}(M)>0$. Moreover, we can take $i>1$ because $b_{1}(M)=b_{m-1}(M)$ by the Poincaré duality. So $0=b_{i-1}\left(S^{m-1}\right)<b_{i}(M)$ and Condition 2 is fulfilled.

If $m=2$ and $M$ is not a rational homology sphere, then $M \neq S^{2}$ is a closed oriented surface. So, $b_{1}(M)>1=b_{0}(N)$ and again Condition 2 is fulfilled.

Condition 4. Let $\mu: N \times S^{1} \rightarrow M$ be a special map of degree $l \neq 0$. Consider the cover map $p: \widetilde{M} \rightarrow M$ such that

$$
\operatorname{Im}\left(p_{*}: \pi_{1}(\widetilde{M}) \rightarrow \pi_{1}(M)\right)=\operatorname{Im}\left(\mu_{*}: \pi_{1}\left(N \times S^{1}\right) \rightarrow \pi_{1}(M)\right) .
$$

Let $\widetilde{\mu}: N \times S^{1} \rightarrow \widetilde{M}$ be a $p$-lifting of $\mu$, i.e., a map such that $\mu=p \circ \widetilde{\mu}$. Clearly, $\operatorname{deg}(\widetilde{\mu}) \operatorname{deg}(p)=\operatorname{deg}(\mu)=l \neq 0$, where deg denotes the degree of a map. Hence the covering $p$ should be finite and $\operatorname{Im} \mu_{*}$ is a finite index subgroup of $\pi_{1}(M)$. Since $\mu$ is special and a map from $\mathcal{N}$ induces the trivial homomorphism $\pi_{1}(N) \rightarrow \pi_{1}(M)$, the composition of homomorphisms

$$
\pi_{1}(N)=\pi_{1}(N \times\{*\}) \stackrel{i_{1 *}}{\longrightarrow} \pi_{1}\left(N \times S^{1}\right) \stackrel{\mu_{*}}{\longrightarrow} \pi_{1}(M)
$$

is the trivial homomorphism. Now, using the formula $\pi_{1}\left(N \times S^{1}\right)=\pi_{1}(N) \oplus \pi_{1}\left(S^{1}\right)$ and the fact that $\operatorname{Im} \mu_{*}$ is a finite index subgroup of $\pi_{1}(M)$, we conclude that

$$
\operatorname{Im}\left(\pi_{1}\left(S^{1}\right)=\pi_{1}\left(\{*\} \times S^{1}\right) \stackrel{i_{2 *}}{\longrightarrow} \pi_{1}\left(N \times S^{1}\right) \stackrel{\mu_{*}}{\longrightarrow} \pi_{1}(M)\right)
$$

is a finite index subgroup of $\pi_{1}(M)$.

If $\operatorname{Im}\left(\mu_{*} \circ i_{2 *}\right)$ is finite, then $\pi_{1}(M)$ is finite. If $\operatorname{Im}\left(\mu_{*} \circ i_{2 *}\right)$ is infinite, then it is isomorphic to $\mathbb{Z}$, and therefore, $\pi_{1}(M)$ contains $\mathbb{Z}$ as a finite index subgroup.

Condition 5. By the Hadamard theorem $\left[8, M\right.$ is a $K\left(\pi_{1}(M), 1\right)$-space. Take a special map $\mu: N \times S^{1} \rightarrow M$.

First, assume that the map

$$
h: S^{1}=\{*\} \times S^{1} \stackrel{i_{2}}{\longrightarrow} N \times S^{1} \stackrel{\mu}{\longrightarrow} M
$$

is homotopy trivial. Since $M$ is a $K\left(\pi_{1}(M), 1\right)$-space, standard obstruction theory arguments show that $\mu$ is homotopic to a map that passes through a projection onto $N^{m-1}$. Thus, $\operatorname{deg} \mu=0$.

Now, assume that the map $h$ is homotopy nontrivial. Clearly, $\pi_{1}(N \times\{*\})$ commutes with $\pi_{1}\left(\{*\} \times S^{1}\right)$ in $\pi_{1}\left(N \times S^{1}\right)$. The Preissman theorem [8] says that all nontrivial Abelian subgroups of $\pi_{1}(M)$ are infinite cyclic. Therefore, $\operatorname{Im} \mu_{*}\left(\pi_{1}\left(N \times S^{1}\right)\right)$ is an infinite cyclic subgroup of $\pi_{1}(M)$ and the homomorphism $\mu_{*}$ has the form $\pi_{1}\left(N \times S^{1}\right) \rightarrow \mathbb{Z} \rightarrow$ $\pi_{1}(M)$. Furthermore, the inclusion $\mathbb{Z} \subset \pi_{1}(M)$ can be induced by a map $\psi: S^{1} \rightarrow M$. Since $M$ is a $K\left(\pi_{1}(M), 1\right)$-space, standard obstruction theory arguments show that $\mu$ : $S^{1} \times N \rightarrow M$ is homotopic to a map $\bar{\mu}: S^{1} \times N \rightarrow S^{1} \stackrel{\psi}{\rightarrow} M$. Thus, $\operatorname{deg} \mu=\operatorname{deg} \bar{\mu}=0$. This completes the proof of Theorem 1.7 .

Remark. It is not always true that $\mathcal{A}(M, \mathcal{N})=\mathbb{Z}$. For example, if $M=T^{2}, N=S^{1}$, and $\mathcal{N}$ consists of maps homotopic to the meridian, then $\mathcal{A}(M, \mathcal{N})=0$. The same is true for $M=S^{2}, N=S^{1}$.

The following theorem says that if the winding number $\operatorname{win}_{p}$ can be defined as the particular case of the classical linking number, then the invariants $\operatorname{win}_{p}, \operatorname{awin}_{p}: \mathcal{N} \backslash \Sigma \rightarrow \mathbb{Z}$ are equal up to an additive constant. Note that, by using Conditions $1-5$ of Theorem 1.9, it is possible to deduce that our affine winding number $\operatorname{awin}_{p}$ is defined for a vast collection of closed $M$ and connected components $\mathcal{N}$ of $C^{\infty}(N, M)$. Recall that for closed 
$M$ the winding number cannot be defined as a particular case of the classical linking number invariant.

1.8. Theorem. Let $M$ be a manifold with $\partial M \neq \varnothing$ or such that it is the interior of a manifold with boundary. Let $\mathcal{N}$ be a connected component of $C^{\infty}(N, M)$ consisting of $f: N \rightarrow M$ with $f_{*}([N])=0 \in H_{*}(M)$. (This is the setup where we can define $\operatorname{win}_{p}: \mathcal{N} \backslash \Sigma \rightarrow \mathbb{Z}$ as the linking number between $f(N)$ and $S^{0}$ consisting of $p$ and a point in a boundary component.) Then $\operatorname{awin}_{p}-\operatorname{win}_{p}: \mathcal{N} \backslash \Sigma \rightarrow \mathbb{Z}$ is a constant function.

Proof. Let $p^{+}$be the 0-dimensional singular cochain $1 \cdot \varphi$, where $\varphi: \Delta^{0} \rightarrow M, \varphi\left(\Delta^{0}\right)=p$. We triangulate $N$ and regard a map $N \rightarrow M$ as a singular chain in $M$. Take $f \in \mathcal{N} \backslash \Sigma$ and recall that $\operatorname{win}_{p}(f)$ is defined as a particular case of the linking number, namely, as the intersection number $\mathfrak{S} \bullet p^{+}$, where $\mathfrak{S}$ is a singular chain with $\partial \mathfrak{S}=f$. Let $F: N \times I \rightarrow M$ be a good homotopy between the preferred map $\varepsilon \in \mathcal{N} \backslash \Sigma$ and $f$. Take a singular chain $\overline{\mathfrak{S}}$ with boundary $\varepsilon$ and consider the triangulation of $N \times I$ such that $\left.F\right|_{N \times 0}$ is equal to $\partial \overline{\mathfrak{S}}$. Then $\mathfrak{S}:=\overline{\mathfrak{S}}+F$ is a singular chain with $\partial \mathfrak{S}=f$. Clearly, $\mathfrak{S} \bullet p^{+}-\overline{\mathfrak{S}} \bullet p^{+}=F \bullet p^{+}$. By Remark 1 $F \bullet p^{+}=\Delta_{\text {awin }_{p}}(F)$. By the definition of $\operatorname{win}_{p}$ we have

$$
F \bullet p^{+}=\mathfrak{S} \bullet p^{+}-\overline{\mathfrak{S}} \bullet p^{+}=\operatorname{win}_{p}(f)-\operatorname{win}_{p}(\varepsilon) .
$$

Hence, $\operatorname{win}_{p}(f)-\operatorname{win}_{p}(\varepsilon)=\Delta_{\operatorname{awin}_{p}}(F)$.

By Theorem 1.7, $\mathcal{A}(M, \mathcal{N})=\mathbb{Z}$ and $q=$ id : $\mathbb{Z} \rightarrow \mathbb{Z}$ for $M$ nonclosed. By the definition of $\operatorname{awin}_{p}$ we have

$$
\operatorname{awin}_{p}(f)=q\left(\Delta_{\operatorname{awin}_{p}}(F)\right)=\Delta_{\operatorname{awin}_{p}}(F)=\operatorname{win}_{p}(f)-\operatorname{win}_{p}(\varepsilon) .
$$

Thus, $\operatorname{awin}_{p}-\operatorname{win}_{p}: \mathcal{N} \backslash \Sigma \rightarrow \mathbb{Z}$ is the constant function $-\operatorname{win}_{p}(\varepsilon)$.

\section{§2. Some APPLICATIONS}

As a first application of our affine winding numbers, we have the following generalization of the Cauchy integral formula.

2.1. Theorem. Let $F^{2}$ be a 2-dimensional surface equipped with a complex structure such that either $F$ is not closed or $F$ has genus exceeding one. Let $f$ be a meromorphic function on $F^{2}$ having poles $\left\{a_{j}\right\}_{j=1}^{k}$ and residues $\operatorname{Res} f\left(a_{j}\right), j=1, \ldots, k$. Let $C_{i}: S^{1} \rightarrow F^{2}$, $i=1,2$, be two homotopic smooth oriented (not necessarily homologous to zero) curves not passing through any of the poles. Let $\operatorname{awin}_{a_{j}}\left(C_{i}\right) \in \mathbb{Z}, i=1,2 ; j=1, \ldots, k$, be the affine winding numbers which are defined for $p=a_{j}$, because condition 0 or condition 1 of Theorem 1.7 is satisfied. Then

$$
\oint_{C_{1}} f d z=\oint_{C_{2}} f d z+2 \pi i\left(\sum_{j=1}^{k} \operatorname{Res} f\left(a_{j}\right)\left(\operatorname{awin}_{a_{j}}\left(C_{1}\right)-\operatorname{awin}_{a_{j}}\left(C_{2}\right)\right)\right) .
$$

This theorem allows one to express the integral of a meromorphic function over a curve through the integral of the function over some specified homotopic curve. The formulation of the classical Cauchy theorem for $F^{2}=\mathbb{C}$ is obtained from Theorem 2.1] by taking $C_{2}$ to be a small curve lying far away from all the poles, so that $\oint_{C_{2}} f d z=0$. In this case, by Theorem 1.8, $\left(\operatorname{awin}_{a_{j}}\left(C_{1}\right)-\operatorname{awin}_{a_{j}}\left(C_{2}\right)\right)$ coincides with the classical winding number $\operatorname{win}_{a_{j}}\left(C_{1}\right)$.

It should be noted that, by statement 2 of Theorem 1.6, the term $\left(\operatorname{awin}_{a_{j}}\left(C_{1}\right)-\right.$ $\left.\operatorname{awin}_{a_{j}}\left(C_{2}\right)\right)$ in (2.1) does not depend on the choice of the preferred map $\varepsilon \in \mathcal{N} \backslash \Sigma$ used to define $\operatorname{awin}_{a_{j}}=\operatorname{awin}_{a_{j}, \varepsilon}$. 
Proof. For nonclosed $F$, the affine winding numbers $\operatorname{awin}_{a_{j}}\left(C_{i}\right)$ are $\mathbb{Z}$-valued, because condition 0 of Theorem 1.7 is fulfilled. For closed $F$ of genus exceeding one, the affine winding numbers $\operatorname{awin}_{a_{j}}\left(C_{i}\right)$ are $\mathbb{Z}$-valued, because $b_{1}\left(S^{1}\right)+b_{0}\left(S^{1}\right)=2<b_{1}(F)$, and hence, Condition 1 of Theorem 1.7 is fulfilled.

Much as for the classical Cauchy Theorem, the proof of Theorem 2.1 boils down to local considerations. Namely, using statement 2 of Theorem 1.6, one shows that both parts of identity (2.1) change in the same way under an elementary homotopy of $C_{1}$ that involves one passage of $C_{1}$ through one of the poles. Since $C_{1}$ is homotopic to $C_{2}$ and the two sides of (2.1) are equal for $C_{1}=C_{2}$, we get identity (2.1).

Applications of $\operatorname{awin}_{p}$ to the study of wave front propagation. Informally speaking, we assume that at a moment of time $T$ something happens at a submanifold of $M^{m}$ and the perturbation caused by this event starts to radiate from the submanifold in all directions in accordance with a propagation law. More accurately, we have a smooth $\operatorname{map} W: N^{m-1} \times[T, \infty) \rightarrow M^{m}$, where $\left.\operatorname{Im} W\right|_{N \times t}$ is thought of as the set of points that the perturbation has reached at time $t$.

In fact, for wave fronts in geometric optics, the map $W$ has special properties. For example, $\left.W\right|_{N \times t}, t \in[T, \infty)$, lifts to a Legendrian submanifold of the unit cotangent bundle of $M$; see Arnold [3. In this paper we do not use any of these properties.

We define the wave front $W(t): N \rightarrow M$ by setting $W(t)(n)=W(n, t), n \in N$, and make the assumption that $W$ is generic, i.e., $p$ is a regular value of $W$ and $(W(t))^{-1}(p)$ has at most one point for each $t \in[T, \infty)$.

We would like to find an estimate from below for the number of times pas $\left(t_{1}, t_{2}\right)$ a wave front $W(t)$ on $M$ passed through the point $p$ between two moments of time $t_{1}$ and $t_{2}$ such that $p \notin \operatorname{Im} W\left(t_{i}\right), i=1,2$. Moreover, we would like this estimate to be computable from the shape of the pairs $\left(W\left(t_{1}\right), p\right)$ and $\left(W\left(t_{2}\right), p\right)$ only, without any knowledge of $W$, the topology of $M$, the time moments $t_{1}, t_{2}$, etc. Clearly, we have $\operatorname{pas}\left(t_{1}, t_{2}\right) \geq\left|\Delta_{\text {awin }_{p}}(F)\right|$, where $F: N \times I \rightarrow M, \quad F(x, t)=W\left(x,\left(t_{2}-t_{1}\right) t+t_{1}\right)$. The difficulty is that we know $W\left(t_{1}\right), W\left(t_{2}\right)$, but we do not know $W(t)$ for $t_{1}<t<t_{2}$, and thus we do not know $F$.

Luckily, statement 2 of Theorem 1.6 says that

$$
q\left(\Delta_{\operatorname{awin}_{p}}(F)\right)=\operatorname{awin}_{p}\left(W\left(t_{2}\right)\right)-\operatorname{awin}_{p}\left(W\left(t_{1}\right)\right)
$$

and that we can take any good homotopy $G$ between $W\left(t_{1}\right)$ and $W\left(t_{2}\right)$ and compute $\operatorname{awin}_{p}\left(W\left(t_{2}\right)\right)-\operatorname{awin}_{p}\left(W\left(t_{1}\right)\right)$ as $q\left(\Delta_{\operatorname{awin}_{p}}(G)\right)$. In particular, if $M, N, \mathcal{N}$ are as in Theorem 1.7. so that $\mathcal{A}(M, \mathcal{N})=\mathbb{Z}$ and $q=\mathrm{id}: \mathbb{Z} \rightarrow \mathbb{Z}$, then $\operatorname{pas}\left(t_{1}, t_{2}\right) \geq$ $\left|\operatorname{awin}_{p}\left(W\left(t_{2}\right)\right)-\operatorname{awin}_{p}\left(W\left(t_{1}\right)\right)\right|$. Thus, in this case we can estimate $\operatorname{pas}\left(t_{1}, t_{2}\right)$ from below knowing the pictures of the front at times $t_{1}$ and $t_{2}$.

2.2. Example. Assume that at the time moments $t_{1}$ and $t_{2}$ the wave front is contained in a chart of $M$. Assume, moreover, that at the time moment $t_{1}$ the picture of the wave front was the one shown in Figure 1 and later at $t_{2}$ it developed to the shape shown in Figure 10. (Figure 1b depicts a sphere that can be obtained from the trivially embedded sphere by passing two times through the point $p$ and by creation of some singularities on the part of the front away from $p$.)

A straightforward calculation gives

$$
\operatorname{awin}_{p}\left(W\left(t_{2}\right)\right)-\operatorname{awin}_{p}\left(W\left(t_{1}\right)\right)=q( \pm 2) \in \mathcal{A}(M, \mathcal{N}),
$$

where the sign depends on the front orientation, which is not shown in Figure 1.

Assume that $M$ is not a rational homology sphere; then Condition 3 of Theorem 1.7 is satisfied. Hence $\mathcal{A}(M, \mathcal{N})=\mathbb{Z}$ and $|q( \pm 2)|=2=\left|\operatorname{awin}_{p}\left(W\left(t_{2}\right)\right)-\operatorname{awin}_{p}\left(W\left(t_{1}\right)\right)\right|$. We conclude that every generic $W$ that changes $W\left(t_{1}\right)$ to $W\left(t_{2}\right)$ involves at least two passages through $p$. 
If $W$ is not generic, it could happen that two branches of the front pass through $p$ simultaneously. However, for nongeneric $W$ we still can conclude that the front passed through $p$ at least once between the two time moments.
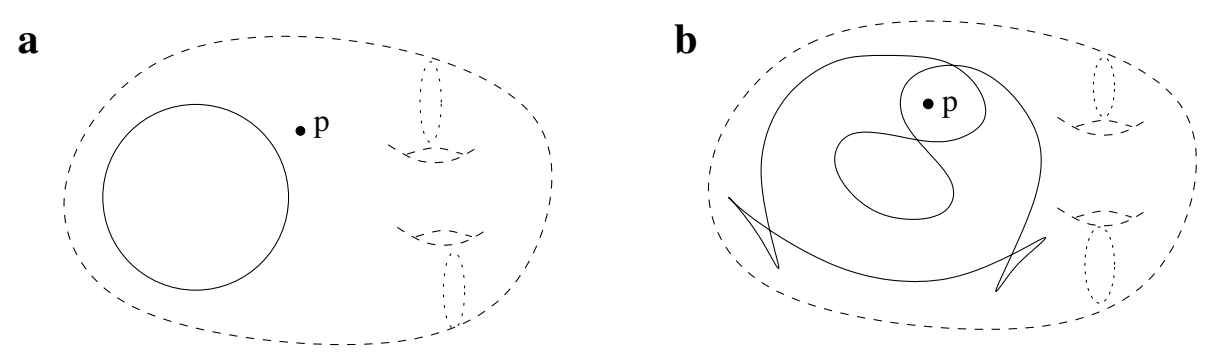

Figure 1

\section{§3. Another generalization of THE Winding NUMBer, AND THE AWIN INVARIANT}

In this section we deal with the case where the point around which we compute the winding number is not stationary but rather moves in $M$. We shall define the affine winding number invariant much as we did before, but the indeterminacy subgroup $\boldsymbol{A}$ will increase.

As before, we fix a connected component $\mathcal{N}$ of $C^{\infty}(N, M)$. We put the discriminant $\widetilde{\Sigma} \subset \mathcal{N} \times M$ to be the set of pairs $(f, x) \in \mathcal{N} \times M$ such that $x \in \operatorname{Im} f$. Note that we do not include into the discriminant the pairs $(f, x)$ such that $f$ is singular in the usual sense but $x \notin \operatorname{Im} f$.

Let $F: N \times I \rightarrow M$ be a smooth map, and let $\gamma: I \rightarrow M$ be a smooth path such that $\gamma(t) \cap F(N \times t)=\varnothing, t=0,1$. By a $C^{\infty}$-small perturbation we may assume that $F$ and $\gamma$ are transverse.

We define $\Phi: N \times I \rightarrow M \times I, \Phi(n, t)=(F(x, t), t)$, and $\Gamma: I \rightarrow M \times I, \Gamma(t)=$ $(\gamma(t), t)$. Since $F$ and $\gamma$ are transverse, we see that $\Phi$ and $\Gamma$ are also transverse. We equip $I=[0,1]$ with the orientation from 0 to 1 and we equip $N \times I$ and $M \times I$ with the product orientations.

3.1. Definition. We define $\Delta_{\mathrm{AWIN}}(F, \gamma) \in \mathbb{Z}$ as the intersection number $\Phi \bullet \Gamma$ of $\Phi$ and $\Gamma$ with respect to the orientations described above.

Note that if $\gamma$ is a constant path, so that $\operatorname{Im} \gamma$ is only one point $p \in M$, then $\Delta_{\text {AWIN }}(F, \gamma)=\Delta_{\operatorname{awin}_{p}}(F)$; see Remark [1.

3.2. Definition $\left(\operatorname{AWIN}(f, x)\right.$-invariant). Fix a pair $(\varepsilon, \rho)=\left(\varepsilon_{\mathcal{N}}, \rho\right) \in \mathcal{N} \times M \backslash \widetilde{\Sigma}$, which should be thought of as a preferred map and a preferred point.

Let $\boldsymbol{A} \subset \mathbb{Z}$ be the subgroup in Definition 1.4 and let $\boldsymbol{B} \subset \mathbb{Z}$ be the subgroup consisting of numbers $\varepsilon_{*}([N]) \bullet a \in \mathbb{Z}$, where $\bullet$ denotes the intersection pairing and $a$ runs over $H_{1}(M)$. Put $\mathcal{B}(M, \mathcal{N})=\mathbb{Z} /(\boldsymbol{A}+\boldsymbol{B})$, and let $\widetilde{q}: \mathbb{Z} \rightarrow \mathcal{B}(M, \mathcal{N})$ be the quotient homomorphism. Clearly, $\mathcal{B}(M, \mathcal{N})$ does not depend on the choice of $(\varepsilon, \rho)$.

Given $(f, x) \in \mathcal{N} \times M \backslash \widetilde{\Sigma}$, we define the affine winding number $\operatorname{AWIN}(f, x)=$ $\operatorname{AWIN}_{\varepsilon, \rho}(f, x) \in \mathcal{B}(M, \mathcal{N})$ by setting

$$
\operatorname{AWIN}(f, x)=\widetilde{q}\left(\Delta_{\mathrm{AWIN}}(F, \gamma)\right) \in \mathcal{B}(M, \mathcal{N}),
$$

where $F: N \times I \rightarrow M$ is a good homotopy between $\varepsilon=\left.F\right|_{N \times 0}$, and $f=\left.F\right|_{N \times 1}$ and $\gamma$ is a smooth path from $\rho$ to $x$ that is transverse to $F$. 
3.3. Theorem. 1. The number $\operatorname{AWIN}(f, x)=\operatorname{AWIN}_{\varepsilon, \rho}(f, x) \in \mathcal{B}(M, \mathcal{N})$ does not depend on the choice of the good homotopy $F$ and of the path $\gamma$ in its definition. Thus, the function

$$
\text { AWIN }: \mathcal{N} \times M \backslash \Sigma \rightarrow \mathcal{B}(M, \mathcal{N})
$$

is well defined.

2. Take $\left(f_{0}, x_{0}\right),\left(f_{1}, x_{1}\right) \in \mathcal{N} \times M \backslash \widetilde{\Sigma}$, a good homotopy $H: N \times I \rightarrow M$ between $f_{0}=\left.H\right|_{N \times 0}$ and $f_{1}=\left.H\right|_{N \times 1}$, and a path $\beta$ from $x_{0}$ to $x_{1}$ that is transverse to H. Then

$$
\operatorname{AWIN}\left(f_{1}, x_{1}\right)-\operatorname{AWIN}\left(f_{0}, x_{0}\right)=\widetilde{q}\left(\Delta_{\mathrm{AWIN}}(H, \beta)\right) .
$$

In particular, AWIN : $\mathcal{N} \times M \backslash \widetilde{\Sigma} \rightarrow \mathcal{B}(M, \mathcal{N})$ is a locally constant function.

3. If we replace $(\varepsilon, \rho)$ by some $\left(\varepsilon^{\prime}, \rho^{\prime}\right) \in \mathcal{N} \times M \backslash \widetilde{\Sigma}$, then

$$
\operatorname{AWIN}_{\varepsilon^{\prime}, \rho^{\prime}}-\operatorname{AWIN}_{\varepsilon, \rho}: \mathcal{N} \times M \backslash \widetilde{\Sigma} \rightarrow \mathcal{B}(M, \mathcal{N})
$$

is the constant function $\operatorname{AWIN}_{\varepsilon^{\prime}, \rho^{\prime}}(\varepsilon, \rho)$. Hence the functions

$$
\operatorname{AWIN}_{\varepsilon^{\prime}, \rho^{\prime}}, \operatorname{AWIN}_{\varepsilon, \rho}: \mathcal{N} \times M \backslash \widetilde{\Sigma} \rightarrow \mathcal{B}(M, \mathcal{N})
$$

are equal up to an additive constant.

Proof. We prove statement 1 . Choose another good homotopy $F^{\prime}$ of $\varepsilon$ to $f$ and another path $\gamma^{\prime}$ from $\rho$ to $x$ that is transverse to $F^{\prime}$. Let $\Phi^{\prime}: N \times I \rightarrow M \times I$ and $\Gamma^{\prime}: I \rightarrow M \times I$ be the corresponding maps. Clearly, it suffices to show that $\Phi \bullet \Gamma-\Phi^{\prime} \bullet \Gamma^{\prime} \in \boldsymbol{A}+\boldsymbol{B}$.

Consider a monotone nondecreasing smooth function $\varphi:[0,1] \rightarrow[0,1]$ such that $\varphi(t)=0$ for $t \in[0,1 / 2]$, and $\varphi(1)=1$. Define good homotopies $\bar{F}, \bar{F}^{\prime}$ via $\bar{F}(x, t)=$ $F(x, \varphi(t)), \bar{F}^{\prime}(x, t)=F^{\prime}(x, \varphi(t))$, and put $\bar{\Phi}, \bar{\Phi}^{\prime}: N \times I \rightarrow M \times I$ to be the corresponding maps. We define $\bar{\gamma}, \bar{\gamma}^{\prime}: I \rightarrow M$ via $\bar{\gamma}(t)=\gamma(1-\varphi(1-t)), \bar{\gamma}^{\prime}(t)=\gamma^{\prime}(1-\varphi(1-t))$ and put $\bar{\Gamma}, \bar{\Gamma}^{\prime}: I \rightarrow M \times I$ to be the corresponding maps.

Since $\Phi$ is homotopic to $\bar{\Phi}$ and $\Gamma$ is homotopic to $\bar{\Gamma}$ modulo boundary, we have $\Phi \bullet$ $\Gamma=\bar{\Phi} \bullet \bar{\Gamma}$, where $\bullet$ is the intersection pairing of the corresponding relative homology classes modulo $\partial(M \times I)$. Similarly, $\Phi^{\prime} \bullet \Gamma^{\prime}=\bar{\Phi}^{\prime} \bullet \bar{\Gamma}^{\prime}$. Thus, it suffices to show that $\bar{\Phi} \bullet \bar{\Gamma}-\bar{\Phi}^{\prime} \bullet \bar{\Gamma}^{\prime} \in \boldsymbol{A}+\boldsymbol{B}$.

Since the maps $\bar{\Phi}, \bar{\Phi}^{\prime}, \bar{\Gamma}, \bar{\Gamma}^{\prime}$ preserve the $I$-coordinate, we have

$$
\begin{aligned}
\bar{\Phi} \bullet \bar{\Gamma}-\bar{\Phi}^{\prime} \bullet \bar{\Gamma}^{\prime}= & \left(\left.\left.\bar{\Phi}\right|_{N \times\left[0, \frac{1}{2}\right]} \bullet \bar{\Gamma}\right|_{\left[0, \frac{1}{2}\right]}+\left.\left.\bar{\Phi}\right|_{N \times\left[\frac{1}{2}, 1\right]} \bullet \bar{\Gamma}\right|_{\left[\frac{1}{2}, 1\right]}\right) \\
& -\left(\left.\left.\bar{\Phi}^{\prime}\right|_{N \times\left[0, \frac{1}{2}\right]} \bullet \bar{\Gamma}^{\prime}\right|_{\left[0, \frac{1}{2}\right]}+\left.\left.\bar{\Phi}^{\prime}\right|_{N \times\left[\frac{1}{2}, 1\right]} \bullet \bar{\Gamma}^{\prime}\right|_{\left[\frac{1}{2}, 1\right]}\right)=\mathfrak{A}+\mathfrak{B},
\end{aligned}
$$

where

$\mathfrak{A}=\left.\left.\bar{\Phi}\right|_{N \times\left[\frac{1}{2}, 1\right]} \bullet \bar{\Gamma}\right|_{\left[\frac{1}{2}, 1\right]}-\left.\left.\bar{\Phi}^{\prime}\right|_{N \times\left[\frac{1}{2}, 1\right]} \bullet \bar{\Gamma}^{\prime}\right|_{\left[\frac{1}{2}, 1\right]}$ and $\mathfrak{B}=\left.\left.\bar{\Phi}\right|_{N \times\left[0, \frac{1}{2}\right]} \bullet \bar{\Gamma}\right|_{\left[0, \frac{1}{2}\right]}-\left.\left.\bar{\Phi}^{\prime}\right|_{N \times\left[0, \frac{1}{2}\right]} \bullet \bar{\Gamma}^{\prime}\right|_{\left[0, \frac{1}{2}\right]}$.

By our choice of $\varphi$, the restrictions $\left.\bar{\gamma}\right|_{\left[\frac{1}{2}, 1\right]},\left.\bar{\gamma}^{\prime}\right|_{\left[\frac{1}{2}, 1\right]}$ are constant paths from $x$ to $x$, and $\left.\bar{F}\right|_{N \times\left[\frac{1}{2}, 1\right]},\left.\bar{F}^{\prime}\right|_{N \times\left[\frac{1}{2}, 1\right]}$ are smooth homotopies of $\varepsilon$ to $f$. Define good homotopies $\widetilde{F}, \widetilde{F}^{\prime}: N \times I \rightarrow M$ of $\varepsilon$ to $f$ via $\widetilde{F}(n, t)=\bar{F}\left(n, \frac{1}{2} t\right), \widetilde{F}^{\prime}(n, t)=\bar{F}^{\prime}\left(n, \frac{1}{2} t\right)$, for $n \in N, t \in I$. By Remark 1 and the observation in Definition 3.1, we get $\mathfrak{A}=\Delta_{\operatorname{awin}_{x}}(\widetilde{F})-\Delta_{\operatorname{awin}_{x}}\left(\widetilde{F}^{\prime}\right)$. By statement 2 of Theorem 1.6, we have

$$
\begin{aligned}
0 & =\operatorname{awin}_{x}(f)-\operatorname{awin}_{x}(f)=q\left(\Delta_{\operatorname{awin}_{x}}(\widetilde{F})\right)-q\left(\Delta_{\operatorname{awin}_{x}}\left(\widetilde{F}^{\prime}\right)\right) \\
& =q(\mathfrak{A}) \in \mathcal{A}(M, \mathcal{N})=\mathbb{Z} / \boldsymbol{A} .
\end{aligned}
$$

Hence, $\mathfrak{A} \in \boldsymbol{A}$. 
By our choice of $\varphi$, the restrictions $\left.\bar{F}\right|_{N \times\left[0, \frac{1}{2}\right]}$ and $\left.\bar{F}^{\prime}\right|_{N \times\left[0, \frac{1}{2}\right]}$ do not move $N$ on $M$, and $\left.\bar{\gamma}\right|_{\left[0, \frac{1}{2}\right]},\left.\bar{\gamma}^{\prime}\right|_{\left[0, \frac{1}{2}\right]}$ are paths from $\rho$ to $x$. Thus, $\mathfrak{B}$ equals the intersection number of $\varepsilon$ and the closed loop $\left.\bar{\gamma}\right|_{\left[0, \frac{1}{2}\right]}\left(\left.\bar{\gamma}^{\prime}\right|_{\left[0, \frac{1}{2}\right]}\right)^{-1}$. Hence, $\mathfrak{B} \in \boldsymbol{B}$.

So, $\mathfrak{A}+\mathfrak{B} \subset \boldsymbol{A}+\boldsymbol{B}$. Now identity (3.1) implies that $\bar{\Phi} \bullet \bar{\Gamma}-\bar{\Phi}^{\prime} \bullet \bar{\Gamma}^{\prime} \in \boldsymbol{A}+\boldsymbol{B}$; we have proved statement 1 of the theorem.

The proofs of statements 2 and 3 are similar to the proofs of the corresponding statements of Theorem 1.6 and therefore are omitted.

The following theorem says that AWIN is a $\mathbb{Z}$-valued invariant for many manifolds $M$ and connected components $\mathcal{N}$ of $C^{\infty}(N, M)$.

3.4. Theorem. The identity $\mathcal{B}(M, \mathcal{N})=\mathbb{Z}$ occurs if $\mathcal{N}$ consists of maps $g$ such that $g_{*}([N]) \in H_{*}(M)$ is a finite order element and at least one of Conditions 0-5 of Theorem 1.7 is satisfied.

Proof. By Theorem [1.7 $\boldsymbol{A}=0 \subset \mathbb{Z}$. Since $\varepsilon_{*}([N]) \in H_{*}(M)$ is an element of finite order, we have $\varepsilon_{*}([N]) \bullet a=0$ for every $a \in H_{1}(M)$. Hence, $\boldsymbol{B}=0 \subset \mathbb{Z}$. Thus, $\mathcal{B}(M, \mathcal{N})=\mathbb{Z} /(\boldsymbol{A}+\boldsymbol{B})=\mathbb{Z}$.

Remark (comparison of AWIN to the winding number that is defined as a particular case of the linking number). Let $M$ be a manifold with $\partial M \neq \varnothing$ or such that $M$ is the interior of a manifold with boundary. Let $\mathcal{N}$ be a connected component of $C^{\infty}(N, M)$ consisting of $f: N \rightarrow M$ with $f_{*}([N])=0 \in H_{*}(M)$. This is the setup where we can define win : $\mathcal{N} \times M \backslash \widetilde{\Sigma} \rightarrow \mathbb{Z}$ as the linking number between $f$ and the map of $S^{0}$ sending one point of $S^{0}$ to $p$ and the other into a chosen boundary component. Then AWIN - win : $\mathcal{N} \times M \backslash \widetilde{\Sigma} \rightarrow \mathbb{Z}$ is a constant function, and hence, the two invariants are equal up to an additive constant. The proof of this fact is similar to the proof of Theorem 1.8 and therefore is omitted.

Remark (AWIN and Viro's winding number). In [20, Viro introduced generalizations of winding numbers to the case of homologous-to-zero immersed curves on a closed surface $F^{2}$ with $\chi\left(F^{2}\right) \neq 0$. Viro's winding numbers are $\mathbb{Q}$-valued and, under regular homotopy, they behave in the same way as the classical winding numbers. However, unlike our affine winding numbers, Viro's winding number around $p \in F^{2}$ changes under a nonregular homotopy of a curve that does not pass through $p$. Hence Viro's winding number does not give rise to a locally constant function on $\mathcal{N} \times F^{2} \backslash \widetilde{\Sigma}$.

Remark (AWIN and wave fronts). The invariant AWIN allows one to estimate from below the number of times a front on $M$ passed through a point that was continuously moving in $M$. Assume that the pair consisting of the trajectory $\alpha:[T,+\infty) \rightarrow M$ of the point and the front propagation $W: N^{m-1} \times[T,+\infty) \rightarrow M^{m}$ is generic, so that $\alpha$ and $W$ are transverse. Choose $t_{1}, t_{2}>T$ such that $\left.\alpha\left(t_{i}\right) \notin \operatorname{Im} W\right|_{N \times t_{i}}, i=1,2$, and define $F: N \times I \rightarrow M$,

$$
F(x, t)=W\left(x,\left(t_{2}-t_{1}\right) t+t_{1}\right), \quad \gamma: I \rightarrow M, \quad \gamma(t)=\alpha\left(\left(t_{2}-t_{1}\right) t+t_{1}\right) .
$$

Clearly, $\left|\Delta_{\text {AWIN }}(F, \gamma)\right|$ estimates from below the number of times the front passes through the moving point between times $t_{1}$ and $t_{2}$.

Put $f_{i}=\left.W\right|_{N \times t_{i}}: N \rightarrow M, i=1,2$. If $M$ and $\left.\mathcal{N} \ni W\right|_{N \times T}$ are such that AWIN is a $\mathbb{Z}$-valued invariant, then $\Delta_{\mathrm{AWIN}}(F, \gamma)=\operatorname{AWIN}\left(f_{2}, \gamma\left(t_{2}\right)\right)-\operatorname{AWIN}\left(f_{1}, \gamma\left(t_{1}\right)\right)$. By statement 2 of Theorem 3.3 the last quantity can be computed by using any good homotopy $H$ of $f_{1}$ to $f_{2}$ and a path $\beta$ from $\gamma\left(t_{1}\right)$ to $\gamma\left(t_{2}\right)$ that is transverse to $H$. This allows us to estimate from below the number of times a front passed through an observable point moving on $M$ between times $t_{1}$ and $t_{2}$. This estimation can be done 
from the snapshots of the front and the point at the two time moments without the knowledge of the front and point movements.

For example, assume that at times $t_{1}$ and $t_{2}$ the front and the observable point were located in a chart of $M$ and were as depicted in Figure 1. Assume that $M$ is not a $\mathbb{Q}$-homology sphere, so that $\mathcal{B}(M, \mathcal{N})=\mathbb{Z}$ by Theorem 3.4. By the above discussion, we see that for generic $(W, \alpha)$ the front passed through an observable moving point at least twice between times $t_{1}$ and $t_{2}$. This conclusion can be made without the knowledge of $W$ and of the trajectory $\alpha$ of the observable point. Much as in the case of a stationary point $p$ discussed in Example 2.2, for nongeneric pairs $(W, \alpha)$ we can still conclude that the front passed through the moving point at least once between the two time moments.

\section{ACKNOWLEDGMENT}

The first author was partially supported by the Walter and Constance Burke Research Initiation Award. The second author was partially supported by NSF, grant 0406311, USA, and by MCyT, projects BFM 2002-00788 and BFM2003-02068/MATE, Spain. His visit to Dartmouth College was supported by funds donated by Edward Shapiro to the Mathematics Department of Dartmouth College.

\section{REFERENCES}

[1] J. E. Andersen, J. Mattes, and N. Reshetikhin, Quantization of the algebra of chord diagrams, Math. Proc. Cambridge Philos. Soc. 124 (1998), no. 3, 451-467. MR.1636568(99m:58040)

[2] _ The Poisson structure on the moduli space of flat connections and chord diagrams, Topology 35 (1996), no. 4, 1069-1083. MR.1404925 (98e:57005)

[3] V. I. Arnol'd, Invariants and perestroikas of fronts on a plane, Trudy Mat. Inst. Steklov. 209 (1995), 14-64; English transl. in Proc. Steklov Inst. Math. MR1422217 (97k:57037)

[4] V. Chernov (Tchernov), Shadows of wave fronts and Arnold-Bennequin type invariants of fronts on surfaces and orbifolds, Differential and Symplectic Topology of Knots and Curves, Amer. Math. Soc. Transl. Ser. 2, vol. 190, Amer. Math. Soc., Providence, RI, 1999, pp. 153-184. MR $1738396(2001 \mathrm{~h}: 57016)$

[5] - Graded Poisson algebras on bordism groups of garlands and their applications, available as a preprint math.GT/0608153 at http://www.arXiv.org (2006).

[6] V. Chernov (Tchernov) and Yu. B. Rudyak, Toward a general theory of linking invariants, Geom. Topol. 9 (2005), 1881-1913; http://www.maths.warwick.ac.uk/gt/GTVol9/paper42.abs.html. MR2175159 (2006g:57050)

[7] Algebraic structures on generalized strings, available as a preprint math.GT/0306140 at http://www.arXiv.org (2003).

[8] M. P. do Carmo, Riemannian geometry, Birkhäuser Boston, Inc., Boston, MA, 1992. MR1138207 (92i:53001)

[9] W. Goldman, Invariant functions on Lie groups and Hamiltonian flows of surface group representations, Invent. Math. 85 (1986), no. 2, 263-302. MR0846929 (87j:32069)

[10] V. Goryunov, Local invariants of mappings of surfaces into three-space, The Arnold-Gelfand Mathematical Seminars, Birkhäuser Boston, Boston, MA, 1997, pp. 223-255. MR1429894 (97m:57040)

[11] U. Kaiser, Link theory in manifolds, Lecture Notes in Math., vol. 1669, Springer-Verlag, Berlin, 1997. MR1479639 (98j:57010)

[12] G. Mikhalkin and M. Polyak, Whitney formula in higher dimensions, J. Differential Geom. 44 (1996), no. 3, 583-594. MR1431007 (97m:57043)

[13] M. Polyak, Shadows of Legendrian links and $J^{+}$-theory of curves, Singularities (Oberwolfach, 1996), Progr. Math., vol. 162, Birkhäuser, Basel, 1998, pp. 435-458. MR1652485 (2000c:57017)

[14] A. Preissman, Quelques propriétés globales des espaces de Riemann, Comment. Math. Helv. 15 (1943), 175-216. MR0010459 (6:20g)

[15] Yu. B. Rudyak, On Thom spectra, orientability, and cobordism, Springer-Verlag, Berlin, 1998. MR:1627486 (99f:55001)

[16] A. Shumakovich, Explicit formulas for the strangeness of a plane curve, Algebra i Analiz 7 (1995), no. 3, 165-199; English transl., St. Petersburg Math. J. 7 (1996), no. 3, 445-472. MR1353494 (98d:57027a) 
[17] Shadow formula for the Vassiliev invariant of degree two, Topology 36 (1997), no. 2, 449-469. MR:1415598 (98g:57015)

[18] V. Turaev, Shadow links and face models of statistical mechanics, J. Differential Geom. 36 (1992), no. 1, 35-74. MR1168981 (93e:57015)

[19] Skein quantization of Poisson algebras of loops on surfaces, Ann. Sci. École Norm. Sup. (4) 24 (1991), no. 6, 635-704. MR.1142906 (94a:57023)

[20] O. Viro, Generic immersions of the circle to surfaces and the complex topology of real algebraic curves, Topology of Real Algebraic Varieties and Related Topics, Amer. Math. Soc. Transl. Ser. 2, vol. 173, Amer. Math. Soc., Providence, RI, 1996, pp. 231-252. MR1384321 (97d:14087)

[21] H. Whitney, On regular closed curves in the plane, Compositio Math. 4 (1937), 276-284. MR 1556973

Department of Mathematics, 6188 Kemeny Hall, Dartmouth College, Hanover, New HampSHIRE 03755

E-mail address: Vladimir.Chernov@dartmouth.edu

Department of Mathematics, University of Florida, 358 Little Hall, Gainesville, Florida 32611-8105

E-mail address: rudyak@math.ufl.edu

Received 14/NOV/2006

Originally published in English 